\title{
The role of clothing in accidental deaths - an ignored problem in developing countries
}

\begin{abstract}
The brain is a very sensitive organ of the body and consequently susceptible to trauma as in cases of road accidents. The authors present a unique case where a woman travelling as a pillion rider on a motorcycle succumbed to cerebral injury. The incident occurred when one end of her long scarf or dupatta got entrapped in the rear wheel of the vehicle and she fell on the road and sustained injuries. This article illustrates the seriousness of wearing loose clothing while riding two wheelers and endangering of lives. In addition, the authors suggest suitable remedial measures to address the ignored aspects of road safety and their importance in preventing morbidity and mortality in developing countries.
\end{abstract}

Keywords: clothing, injury, accident, scarf, dupatta

\author{
Volume I Issue 4 - 2015 \\ Satinder Pal Singh,' Surinder Pal Singh, ${ }^{2}$ \\ Sumeet Kaur, ${ }^{3}$ Dalbir Singh, ${ }^{4}$ Kumaran $M^{5}$ \\ 'Department of Forensic Medicine and Toxicology, Government \\ Medical College and Hospital, India \\ ${ }^{2}$ Department of Tuberculosis and Chest Diseases, Government \\ Medical College, India \\ ${ }^{3}$ Department of Biochemistry, Government Medical College and \\ Hospital, India \\ ${ }^{4}$ Department of Forensic Medicine, Post Graduate Institute of \\ Medical Education and Research, India \\ ${ }^{5}$ Department of Forensic Medicine, Sri Manakula Vinayagar \\ Medical College and Hospital, India
}

Correspondence: Satinder Pal Singh, Department of Forensic Medicine and Toxicology, Government Medical College and Hospital, Chandigarh, India, Tel +9l 9988008723.

Email spsingh9988@yahoo.co.in

Received: October 24, 2015 | Published: November 13, 2015

\section{Introduction}

Every year, thousands of people lose their lives due to road accidents. The shocking state of road safety is a cause of concern as it not only devastates the family of the deceased, but also puts enormous financial burden on the national resources. The road accident deaths put a burden of more than US \$518 on the international economy. ${ }^{1}$ India reports one accident every minute and one death every four minutes due to road accidents. ${ }^{2}$ Most of these accidents occur as a result of collision between vehicles or structures present on the side of roads. However, some cases have also been reported where the accidents take place due to entrapment of loose clothing in wheels of the vehicle and consequent fall on roads. The authors present an uncommon case from this part of India where a female lost life due to her negligence.

\section{Case report}

A 45 years old lady was travelling as a pillion rider on motorcycle. Suddenly her dupatta got entangled in the spokes of the rear tyre of the motorcycle and she fell on the road and sustained serious injuries (Figure 1). She was rushed to a local hospital from where she was referred to this institute as it is one of the leading tertiary care hospitals in this region of India. However, in spite of the all the efforts of the doctors and hospital staff, she could not be saved and succumbed to her injuries two day after the incident and the post mortem conducted at 14 hours after death.

\section{Autopsy findings}

The Indian law require medicolegal postmortem in each and every case of unnatural death and hence dead body was shifted to the mortuary of the institute. On the external examination, the length of the dead body was $151 \mathrm{cms}$ and weight $59 \mathrm{kgs}$. The rigor mortis was present all over the body. Postmortem staining was present on the back of body except pressure areas and was fixed. A reddish brown abrasion of $10 \mathrm{cmsx} 1 \mathrm{~cm}$ was present obliquely over the front of neck, $1.5 \mathrm{~cm}$ below the thyroid cartilage and moved obliquely backwards and upwards in the direction of the right lateral side of neck and ended $4 \mathrm{~cm}$ below the right pinna (Figure 2).

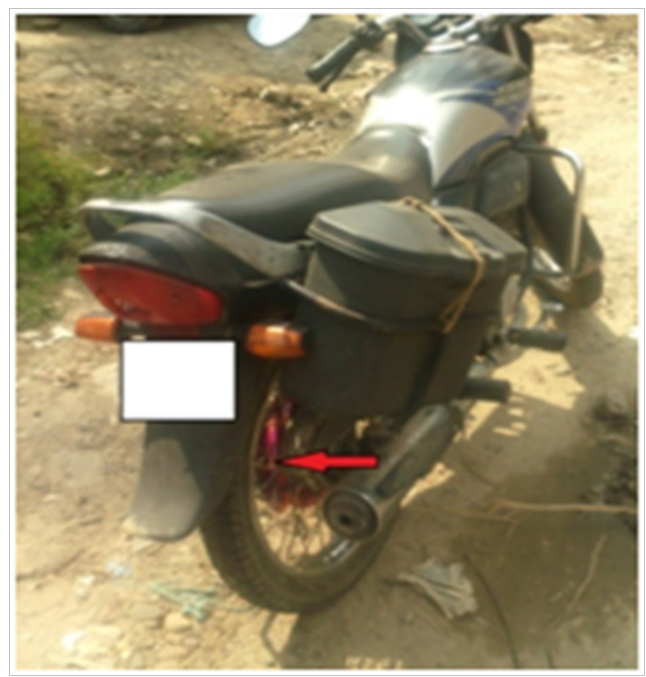

Figure I The motorcycle and the entangled dupatta in the rear tyre.

I. On dissection of the neck, however no significant findings were observed. No intimal tears were present on the carotid artery.

II. A lacerated wound of $8 \mathrm{cmsx} 2.3 \mathrm{cms}$ was present obliquely on the occipital region of the head at the level of occipital protuberance. Clotted blood was present. 
III. Multiple bluish contusions of varying sizes and shapes were present on the front of chest and both breasts.

IV. A bluish contusion of $4 \mathrm{cms} \times 3 \mathrm{cms}$ was present on the anterio lateral aspect of left thigh, $8 \mathrm{~cm}$ below the right anterior superior iliac spine.

V. A bluish contusion of $3 \mathrm{~cm} \mathrm{x} 4 \mathrm{~cm}$ was present on the front of left knee.

VI. A bluish contusion of $3 \mathrm{~cm} \times 2 \mathrm{~cm}$ was present on the front of right knee.

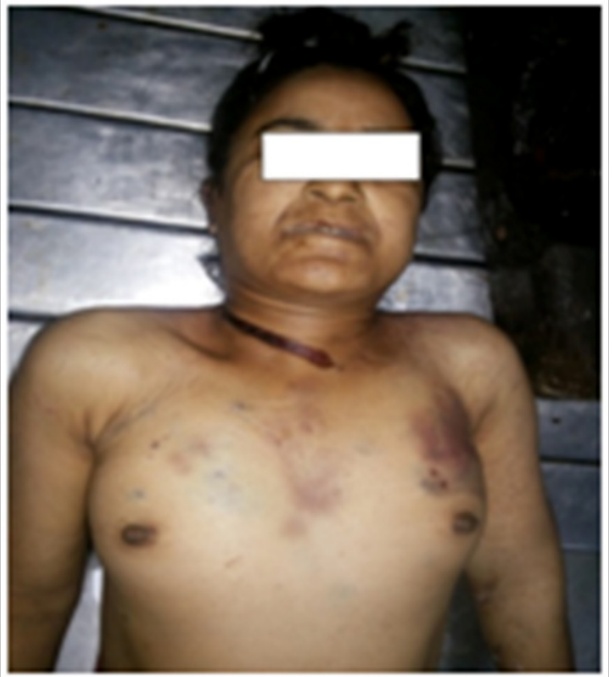

Figure 2 Injuries on the body of deceased.

There was subaponeurotic hemorrhage present over the occipital and both parietal regions. On internal examination, the brain weighed 1240 grams. On dissection, the occipital region on the right side was lacerated. The subdural and subarachanoid hemorrhages were present over both frontal, both parietal and right temporal regions (Figure 3). The base of brain contained about $90 \mathrm{cc}$ of fluid and clotted blood. The cause of death in this case was given as cerebral trauma as a result of accidental fall which was sufficient to cause death in the ordinary course of nature.

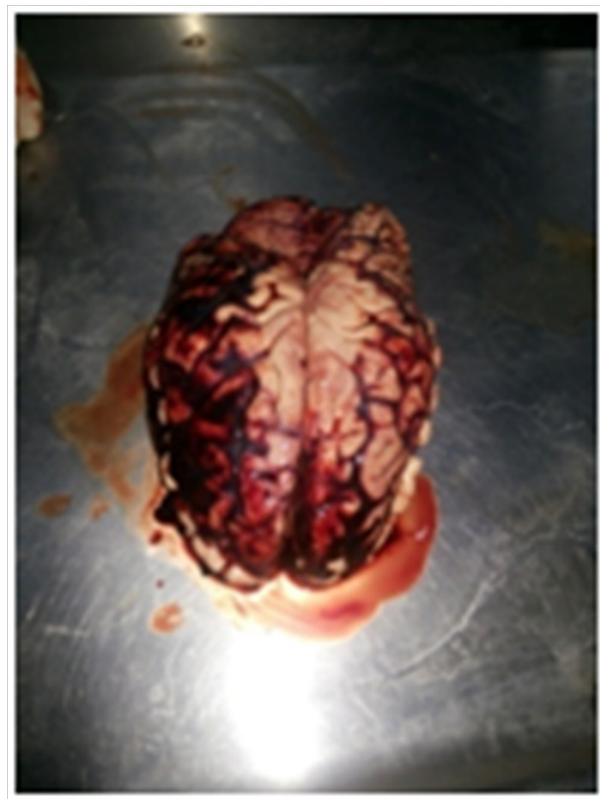

Figure 3 The findings in the brain.

\section{Discussion}

The dupatta is a long scarf and is worn over the head by women. It is a loose piece of cloth of about 2 to $2.5 \mathrm{mts}$ and forms a part of traditional attire in India. The loose clothing has a greater chance to get entangled and become a means of injuries. The association of such loose clothing in sustaining injuries has been documented in literature. ${ }^{3-6}$ In addition, the absence of safety gears is also known to increase the incidence of severe injuries. This issue is of particular significance to Indian women as it is not mandatory for them to wear helmets in many parts of the country. Consequently, the safety issues largely remain unaddressed. In the past, women members of a particular community in North India protested against making the wearing of helmet compulsory as they tie their hair over the head and cannot thus wear helmets. As religious freedom is a big issue in India, women were 'spared' of the compulsion of wearing helmets. But many studies have suggested that the absence of helmets is likely to increase the morbidity and mortality from head injuries. ${ }^{7,8}$ In most of these cases, head injury remains the leading cause of death in two wheeler accidents. ${ }^{9}$ The authors believe and advice that those who do not tie hair over the head should be encouraged to wear helmets and all road safety laws should be strictly enforced. The enforcement of laws has not been up to the mark in India. ${ }^{10}$ In the present case, injuries were sustained as the dupatta of the victim got entangled in the spokes of rear tyre of the motorcycle and she fell on the road and lost her life due to consequent head injury. Such injuries and deaths can be prevented by avoiding loose clothing while driving. Additionally, a 'cover' or 'guard' over the spokes of the wheel can be provided to prevent such mishaps.

\section{Acknowledgments}

None.

\section{Conflicts of interest}

The author declares that there are no conflicts of interest.

\section{References}

1. Zhang X, Xiang H, Jing R, et al. Road traffic injuries in People's Republic of China, 1951-2008. Traffic Inj Prev. 2011;12(6):614-620.

2. United Nations. Economic and Social Commission Asia and the Pacific. Status paper on road safety in India. 2013.

3. Meena MC, Nidhi Sachedeva, Mukta Rani, et al. Accidents are caused, they don't happen. Scandinavian Journal of Forensic Science. 2013;19(1):7-9.

4. Krishan Vij. The Textbook of Forensic Medicine - Principles and Practice. Anil Aggrawal's Internet Journal of Forensic Medicine and Toxicology. 2001;2(2):14.

5. Siddiqui AA, Shamim MS, Jooma R, et al. Long scarf injuries. $J$ Coll Physicians Surg Pak. 2006;16(2):152-153.

6. Deidiker RD. Accidental ligature strangulation due to a roller type massager device. Am J Forensic Med Pathol. 1999;20(4):354-356.

7. Lin MR, Kraus JF. A review of risk factors and patterns of motorcycle injuries. Accid Anal Prev. 2009;41(4):710-722.

8. Gururaj G. Epidemiology of Head Injuries-Project report. NIMHANS, KSCST, Bangalore, India. 1993.

9. Sharma BR, Harish D, Gauri Singh, et al. Patterns of fatal head injury in road traffic accidents. Bahrain Medical Bulletin. 2003;25(1):22-25.

10. World Health Organization (WHO). Global status report on road safety 2013-Supporting a decade of action. World Health Organization technical report series, Geneva. 2013. 